\title{
Acral Peeling Skin Syndrome Resembling Epidermolysis Bullosa Simplex in a 10-Month-Old Boy
}

\author{
$\begin{array}{llll}\text { S. Kavaklieva } & \text { I. Yordanova } & \text { L. Bruckner-Tuderman } & \text { C. } \text { Has }^{c}\end{array}$ \\ ${ }^{a}$ Royal Lancaster Infirmary, University Hospitals of Morecambe Bay NHS Foundation Trust, \\ Lancaster, UK; ${ }^{b}$ Department of Dermatology and Venereology, Medical University Pleven, \\ Pleven, Bulgaria; 'Department of Dermatology, University Medical Center Freiburg, \\ Freiburg, Germany
}

\section{Key Words}

Acral peeling skin syndrome $\cdot$ TGM5 gene $\cdot$ CSTA gene

\begin{abstract}
The acral peeling skin syndrome (APSS) is a rare autosomal recessive disorder clinically characterized by asymptomatic desquamation of the skin limited to the hands and feet and histologically by cleavage at the stratum granulosum and stratum corneum level [Kiritsi et al.: J Invest Dermatol 2010;130:1741-1746]. We report on a 10-month-old boy with a history of skin peeling limited to the hands and feet since 2 months of age. Clinical examination revealed erythematous erosions with peripheral desquamation and flaccid blisters. DNA mutation analysis detected two heterozygous TGM5 mutations: c.2T>C, p.M1T in exon 1 and c.337G > T, p.G113C in exon 3 in keeping with the diagnosis of APSS. The clinical presentation of APSS alone might be confusing and strongly resemble epidermolysis bullosa simplex making the differential diagnosis difficult.

(C) 2013 S. Karger AG, Basel
\end{abstract}

\section{Introduction}

The acral peeling skin syndrome (APSS) is a rare autosomal recessive disorder clinically characterized by asymptomatic desquamation of the skin limited to the hands and feet and histologically by cleavage at stratum granulosum and stratum corneum level [1]. As the body of literature is growing, it becomes apparent that the condition is determined by mutations in the TGM5 gene encoding transglutaminase 5 or in the CSTA gene encoding cystatin A [2]. Moreover, it was reported that in children, APSS manifests clinically with blisters and 
Kavaklieva et al.: Acral Peeling Skin Syndrome Resembling Epidermolysis Bullosa Simplex in a 10-Month-Old Boy

erosions on the palms and soles and is not limited to the dorsal aspect of the hands and feet [1]. It was suggested that the clinical presentation of APSS might be confusing and strongly resemble epidermolysis bullosa simplex (EBS) making the differential diagnosis difficult.

\section{Case Report}

Here, we report on a 10-month-old boy with a history of skin peeling limited to the hands and feet since 2 months of age.His mother noticed worsening of the condition with heat and humidity but not by mechanical trauma. The baby was born from a normal pregnancy and delivery and has non-consanguineous parents. The family history was negative. Clinical examination showed erythematous erosions with desquamation at the periphery on both palms and soles as well as a flaccid blister on the finger of the right hand (fig. 1, fig. 2). The mycological examination of the palms and soles was negative. The baby's mother refused a skin biopsy from the edge of a fresh blister for histological examination. DNA mutation analysis was performed in the 10-month-old boy as well as in his mother and father. From the extracted DNA of the index case, the entire coding region and the flanking intronic regions of the TGM5 gene (Genbank NM_201631.3, NC_000015.9) were analyzed using the PCR followed by DNA sequencing as described [3]. In the parents, only the coding exons 1 and 3, and the flanking intronic regions were analysed. In the index patient, we detected two heterozygous TGM5 mutations: c.2T>C, p.M1T in exon 1 and c.337G $>\mathrm{T}$, p.G113C in exon 3. The father is a heterozygous carrier of the mutation c.2T $>$ C, p.M1T and the mother is a heterozygous carrier of the mutation c.337G>T, p.G113C (fig. 3). No other mutations were disclosed in the analyzed regions.

The baby's parents have been advised to communicate with a human geneticist or a genetic counselor. They were informed about preventive measurements: avoid heat and humidity by using shoes made from breathing textile materials and leather as well as to use topical emollient application.

\section{Discussion}

PSS, which was first described in 1921, is a rare autosomal recessive genodermatosis characterized by shedding of the cornified layer of the skin [4]. It was broadly classified into localized and generalized forms. The APSS is considered to be a localized variant (APSS MIM 609796) [5]. Originally, it was described as peeling of the skin limited to the dorsa of the hands and provoked by mechanical trauma and humidity [3]. Ilknur et al. [6] have also described cases in which acral and generalized skin peeling occur together. Ultrastructural and light microscopic studies in both forms of the disorder show the same level of blistering in the epidermis at the stratum granulosum and stratum corneum junction. Genetically, generalized and localized acral forms seem to be heterogeneous. The molecular basis of APSS was elucidated in 2005 by Cassidy et al. [3] who localized the genetic defect on chromosome 15 (at 15q15.2). TGM5 is expressed in the epidermal granular cells where it cross-links a variety of structural proteins in the terminal differentiation of the epidermis to form the cornified cell envelope. Recently, Pavlovic et al. [7] reported a new APSS pedigree in which they failed to disclose TGM5 gene mutations. Subsequently, using whole exome sequencing, CSTA mutations were identified [2]. Originally, cystatin A was associated with another rare genodermatosis, exfoliative ichthyosis, and therefore the clinical overlap/distinction between these two entities remains to be established [8]. Thus, APSS 
Kavaklieva et al.: Acral Peeling Skin Syndrome Resembling Epidermolysis Bullosa Simplex in a 10-Month-Old Boy

represents a clinically and genetically heterogeneous disorder. EBS is clinically very similar to APSS showing blisters and erosions on the palms and soles. Although the blisters are also localized intraepidermally, they are located in the basal layer and usually occur after mechanical traumas. In most cases, mutations in the genes for keratin 5 and 14 are found [1].

In conclusion, we present a case of genetically confirmed APSS, which clinically resembles EBS. Both mutations, c.337G > T, p.G113C and c.2T >C, p.M1T have been published before in the literature in patients with APSS $[3,9]$. The parents are both heterozygous carriers of the mutations. We consider that APSS is not so rare and that often APSS cases are diagnosed as EBS cases, especially when no DNA mutation analysis is performed. Particularly in little babies, it is important not to underestimate the diagnosis of APSS because of the different outcome of the two diseases. In older children and in the adult APSS patients, peeling of the skin was the most prominent symptom, whereas blistering almost ceased. It is also essential to inform parents regarding the autosomal recessive inheritance of APSS.

\section{Disclosure Statement}

The authors have no conflicts of interest to disclose.

\section{References}

1 Kiritsi D, Cosgarea I, Franzke CW, Schumann H, Oji V, Kohlhase J, Bruckner-Tuderman L, Has C: Acral peeling skin syndrome with TGM5 gene mutations may resemble epidermolysis bullosa simplex in young individuals. J Invest Dermatol 2010;130:1741-1746.

2 Krunic AL, Stone KL, Simpson MA, McGrath JA: Acral peeling skin syndrome resulting from a homozygous nonsense mutation in the CSTA gene encoding cystatin A. Pediatr Dermatol 2013, E-pub ahead of print.

3 Cassidy AJ, van Steensel MA, Steijlen PM, van Geel M, van der Velden J, Morley SM, Terrinoni A, Melino G, Candi E, McLean WH: A homozygous missense mutation in TGM5 abolishes epidermal transglutaminase 5 activity and causes acral peeling skin syndrome. Am J Hum Genet 2005;77:909-917.

4 Fox H: Skin shedding (keratolysis exfoliativa congenita): report of a case. Arch Dermatol Syph 1921;3:202.

5 Kharfi M, El Fekih N, Ammar D, Jaafoura H, Schwonbeck S, van Steensel MA, Fazaa B, Kamoun MR, Fischer J: A missense mutation in TGM5 causes acral peeling skin syndrome in a Tunisian family. J Invest Dermatol 2009;129:2512-2515.

6 Ilknur T, Demirtaolu M, Akarsu S, Lebe B, Güne AT, Ozkan S: Peeling skin syndrome. Eur J Dermatol 2006;16:287-289.

7 Pavlovic S, Krunic AL, Bulj TK, Medenica MM, Fong K, Arita K, McGrath JA: Acral peeling skin syndrome: a clinically and genetically heterogeneous disorder. Pediatr Dermatol 2012;29:258-263.

8 Blaydon DC, Nitoiu D, Eckl KM, Cabral RM, Bland P, Hausser I, van Heel DA, Rajpopat S, Fischer J, Oji V, Zvulunov A, Traupe H, Hennies HC, Kelsell DP: Mutations in CSTA, encoding cystatin A, underlie exfoliative ichthyosis and reveal a role for this protease inhibitor in cell-cell adhesion. Am J Hum Genet 2011;89:564571.

9 Pigors M, Kiritsi D, Cobzaru C, Schwieger-Briel A, Suárez J, Faletra F, Aho H, Mäkelä L, Kern JS, BrucknerTuderman L, Has C: TGM5 mutations impact epidermal differentiation in acral peeling skin syndrome. J Invest Dermatol 2012;132:2422-2429. 
Kavaklieva et al.: Acral Peeling Skin Syndrome Resembling Epidermolysis Bullosa Simplex in a 10-Month-Old Boy

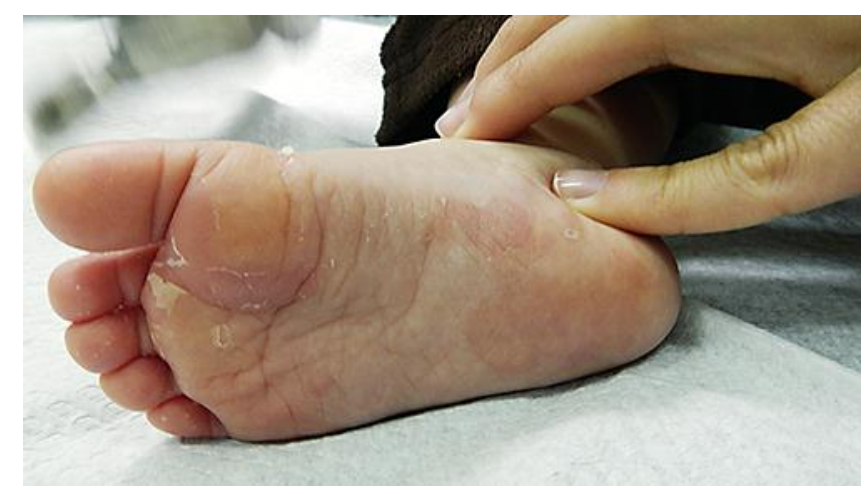

Fig. 1. Residual erythema and skin peeling on the plantar aspect of the feet.

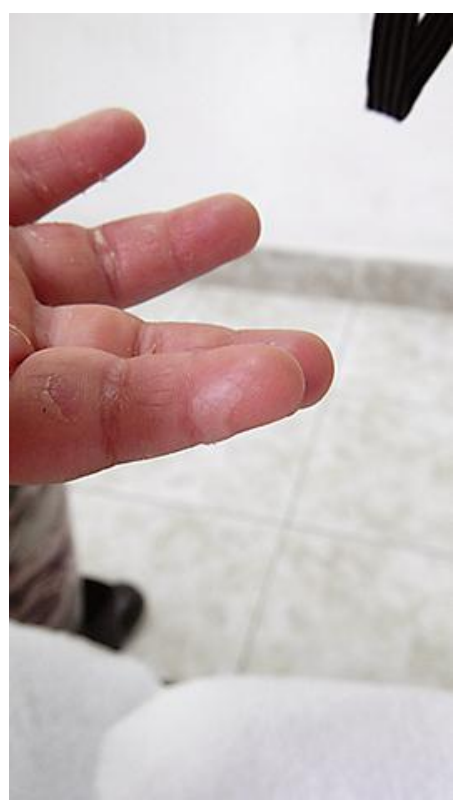

Fig. 2. Flaccid blister on the palmar aspect of the right index finger. 
Kavaklieva et al.: Acral Peeling Skin Syndrome Resembling Epidermolysis Bullosa

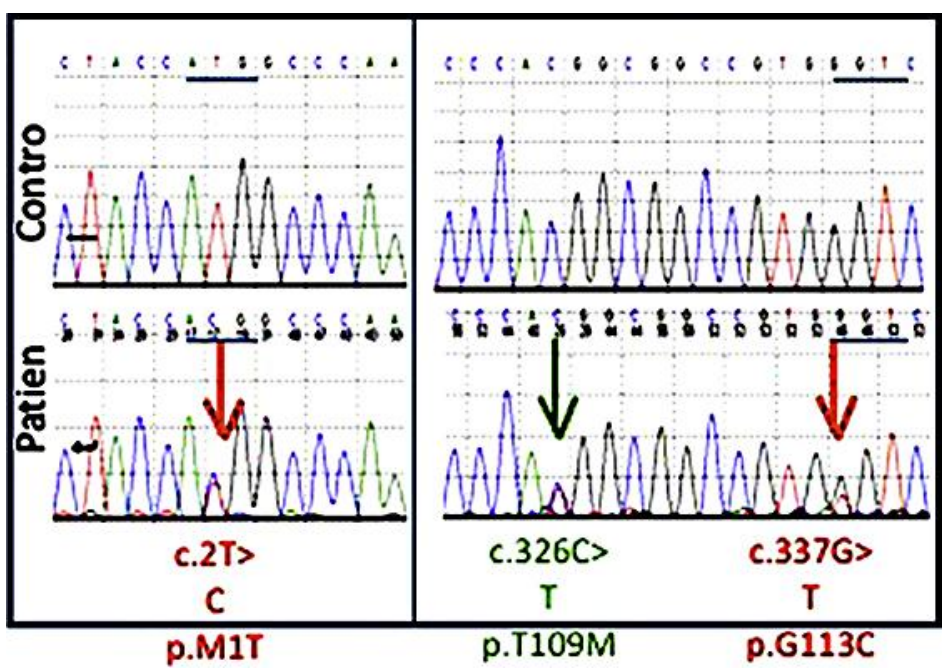

Fig. 3. TGM5 mutations in the index case. Partial sequences of exons 1 (left) and 3 (right) of TGM5 in a control and index case are shown. Mutations are indicated by red arrows and the corresponding codons are underlined. The green arrow points to the single nucleotide polymorphism p.T109M (rs113463533). 